\title{
Robotic Head and Neck Surgery: History, Technical Evolution and the Future
}

\author{
George Garas ${ }^{a, b}$ Asit Arora c \\ aDepartment of Otorhinolaryngology and Head and Neck Surgery, St. Mary's Hospital, \\ Imperial College Healthcare NHS Trust, London, UK; ${ }^{b}$ Department of Surgical Research \\ and Innovation, The Royal College of Surgeons of England, London, UK; ' Department of \\ Otorhinolaryngology and Head and Neck Surgery, Guy's and St. Thomas' NHS Foundation \\ Trust, London, UK
}

\section{Keywords}

Robotics · Transoral surgery · Thyroid surgery · Parathyroid surgery · Evolution · Technology · Innovation · Safety · Evidence

\begin{abstract}
The first application of robotic technology in surgery was described in 1985 when a robot was used to define the trajectory for a stereotactic brain biopsy. Following its successful application in a variety of surgical operations, the da Vinci ${ }^{\circledR}$ robot, the most widely used surgical robot at present, made its clinical debut in otorhinolaryngology and head and neck surgery in 2005 when the first transoral robotic surgery (TORS) resections of base of tongue neoplasms were reported. Subsequently, the indications for TORS rapidly expanded, and they now include tumours of the oropharynx, hypopharynx, parapharyngeal space, and supraglottic lar$y n x$, as well as obstructive sleep apnoea (OSA). The da Vinci ${ }^{\circledR}$ robot has also been successfully used for scarless-in-the-neck thyroidectomy and parathyroidectomy. At present, the main barrier to the wider uptake of robotic surgery is the prohibitive cost of the da $\mathrm{Vinci}^{\circledR}$ robotic system. Several novel, flexible surgical robots are currently being developed that are likely to not only enhance patient safety and expand current indications but also drive down costs, thus making this innovation more widely available. Future directions relate to overlay technology through augmented reality/AR that allows real-time image-guidance, miniaturisation (nanorobots), and the development of autonomous robots.

(C) 2018 S. Karger AG, Basel
\end{abstract}


Fig. 1. The key surgical robots and associated operations that they have facilitated, listed chronologically. TURP, transurethral resection of the prostate; $\mathrm{CABG}$, coronary artery bypass grafting.

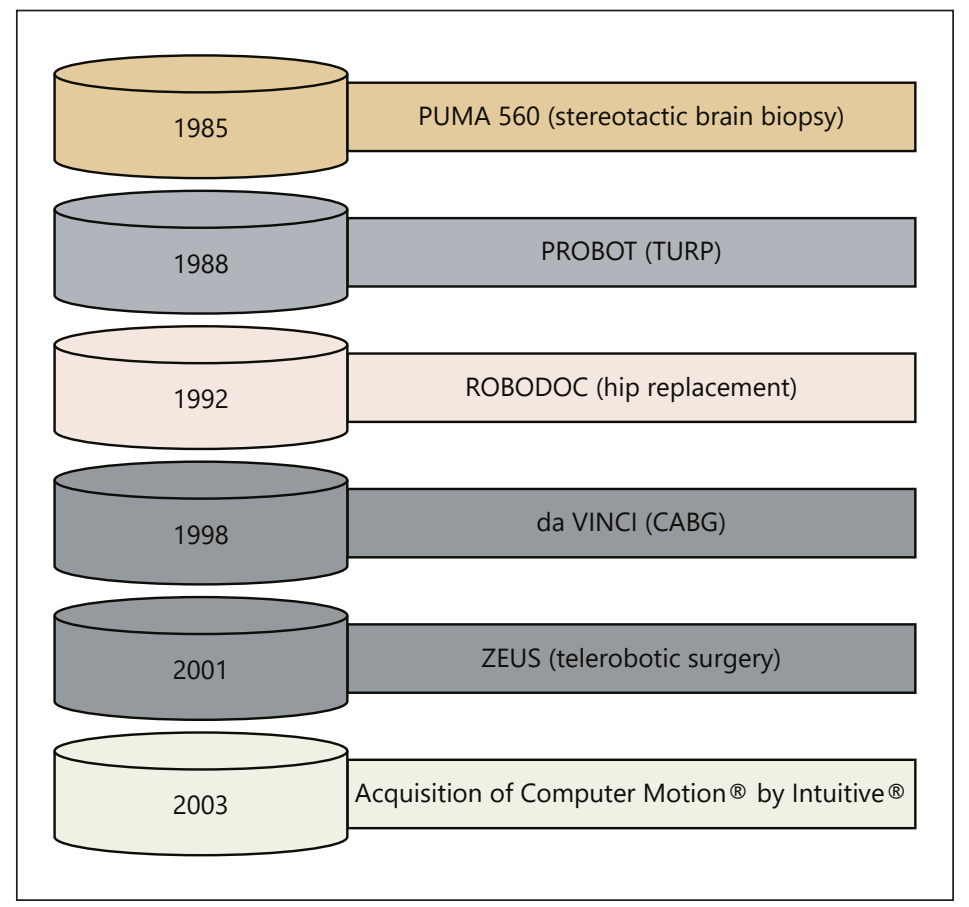

\section{History: from Leonardo da Vinci to the da Vinci ${ }^{\circledR}$ Surgical Robot}

Leonardo da Vinci designed the first robot in 1495. This was a mechanical, armoured knight used for the purposes of entertaining royalty [1]. However, it was not until centuries later that robots first appeared in popular science fiction [2]. The word "robot" originates from the Czech word "robota" meaning labourer, and was first coined by the Czech writer Karel Capek in his play Rossum's Universal Robots in 1921 [3]. It was this work that first introduced the concept of robots in lay people's minds.

The most significant advances in robotic technology were attained as a result of research conducted by the US Military and the National Aeronautics and Space Administration (NASA) in the 1970s and 1980s. Their aim was to design and produce robots that could be remotely controlled (telerobotics) without requiring the presence of the surgeon or operator on the battle field or in outer space [4]. Another military robotic application includes drones, i.e., remotely controlled unmanned aircraft systems. Drones can be fitted with cameras and/or missiles, in order to carry out surveillance and/or offensive missions, respectively, in war zones located thousands of miles away without putting the "pilot" at risk [5].

Nowadays, robots are widely incorporated in industry as well as in everyday life. Examples from industry include the automated robotic arms used in car manufacturing (MercedesBenz, Inc., Stuttgart, Germany) as well as in packaging and labeling products in factories. In everyday life, robots have been designed to assist the elderly with the activities of daily living (Kompai ${ }^{\circledR}$ robot, Robosoft, Bidart, France), and drones are also used for civilian purposes such as delivering commodities purchased online (Amazon.com, Inc., Seattle, WA, USA).

The use of robotic technology in surgery was first described in the 1980s. Figure 1 summarises the key surgical robots and associated operations that they facilitated, in chronological order.

In 1985, the PUMA $5600^{\circledR}$ robot (Advance Research \& Robotics, Inc., Oxford, CT, USA) was used to perform the first robotic-assisted CT-guided stereotactic brain biopsy [6] whilst in 
1988, the PROBOT ${ }^{\circledR}$ (Imperial College London, London, UK) was used to perform the first robotic-assisted transurethral resection of the prostate (TURP) [7]. Several surgical robots followed, including the ROBODOC ${ }^{\circledR}$ (Integrated Surgical Systems, Inc., Sacramento, CA, USA), the first US FDA-approved robot for hip replacement [8], and the ZEUS ${ }^{\circledR}$ robotic surgical system (Computer Motion, Inc., Santa Barbara, CA, USA) used to perform the first telerobotic operation in 2001, a trans-Atlantic robotic-assisted laparoscopic cholecystectomy performed in Strasbourg, France, by a surgeon located in New York, NY, USA [9].

In 2003, following several lawsuits for alleged patent infringement by Computer Motion, Inc. against Intuitive Surgical, Inc. (Sunnyvale, CA, USA), another California-based surgical robotics company and manufacturer of the da Vinci ${ }^{\circledR}$ surgical robot, Intuitive Surgical, Inc. acquired Computer Motion, Inc. and phased out ZEUS ${ }^{\circledR}$ in favour of the da Vinci ${ }^{\circledR}$ surgical robot [10]. Since then, this device has essentially monopolised the robotic surgery market worldwide [11].

The da Vinci ${ }^{\circledR}$ surgical robot constitutes the most commercially successful robotic surgical platform to date, having sold over 5,000 units worldwide. Although originally designed for endoscopic cardiac surgery (closed-chest coronary artery bypass grafting), its main application relates to robotic prostatectomy. Following its introduction in 1997, FDA approval was obtained in 2000 [12]. It has since been used in a variety of surgical procedures, primarily relating to body cavities such as the abdomen, pelvis, and thorax, facilitating conventional laparoscopic and thoracoscopic surgery, respectively [12].

Apart from the market monopoly achieved via the acquisition and closure of key competitors by Intuitive Surgical, Inc. as well as their intense marketing and patenting of several robotic components [13], the reason why the da Vinci ${ }^{\circledR}$ surgical robot has been commercially successful is due to the several advantages it offers over conventional endoscopic surgery [14]. These are summarised in Figure 2.

\section{Transoral Robotic Surgery}

Having received FDA approval for numerous procedures in a variety of surgical specialties, including general surgery, urology, cardiothoracic surgery, and gynaecology, the da Vinci ${ }^{\circledR}$ surgical robot made its clinical debut in otorhinolaryngology and head and neck surgery in 2005 at the University of Pennsylvania at the hands of Profs. Bert O'Malley and Gregory Weinstein [15]. This is when the term "TORS" was first coined (as an acronym for TransOral Robotic Surgery).

TORS capitalised on the presence of the oral cavity as an access point for natural orifice transluminal endoscopic surgery (NOTES), thus providing access to the pharynx, parapharyngeal space, and larynx, without the morbidity of cervical incisions or the limitations associated with previously described transoral approaches, namely transoral laser microsurgery (TLM) [16].

Initial indications for TORS involved base of tongue neoplasms, but, with increasing experience combined with preclinical studies on animals and cadavers, these rapidly expanded [17]. Currently, TORS constitutes a valuable treatment modality for tumours of the oropharynx (including advanced oropharyngeal carcinoma) [18], hypopharynx [19], parapharyngeal space [20], and supraglottic larynx [21]. More recently, it has been deployed in the management of carcinoma of unknown primary tumours [22] as well as for transoral robotic total laryngectomy [23]. In addition to malignant disease, TORS has been shown to be a valuable treatment for obstructive sleep apnoea (OSA) due to the excellent access it offers, and it represents an ideal tool for targeted multilevel surgery in patients who do not tolerate continuous positive airway pressure (CPAP) [24-26]. 


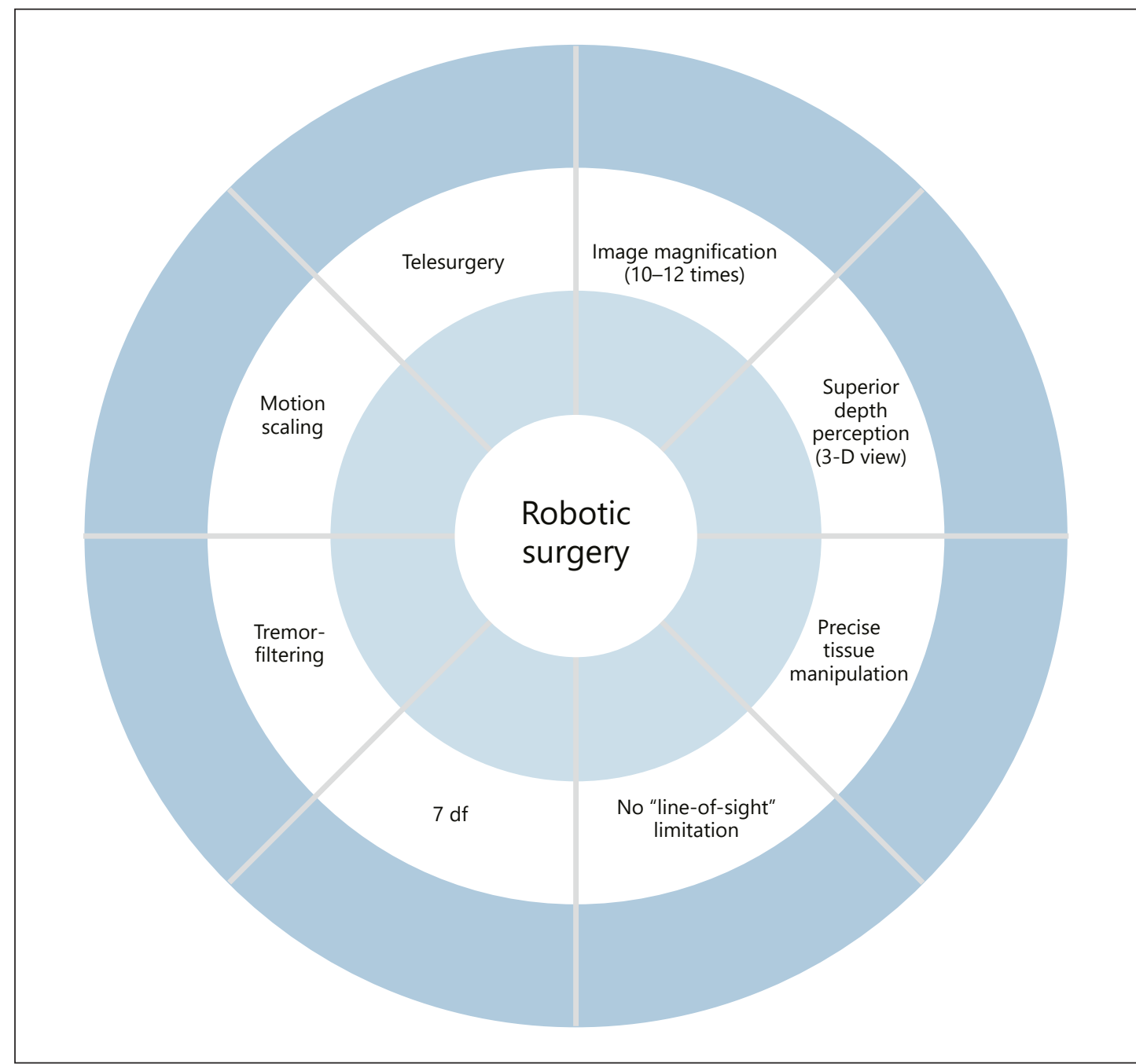

Fig. 2. Advantages of the da Vinci ${ }^{\circledR}$ surgical robot over conventional endoscopic head and neck surgery.

In just over a decade, TORS has evolved from a proof-of-concept to a standard-of-care in high-volume robotic centres, having obtained FDA approval for both benign and malignant diseases of the head and neck in 2009 [27]. However, the evidence for TORS remains limited to case series and retrospective studies $[15,18,20]$. To address this, there are 3 multi-centre randomised controlled trials (RCTs) currently in the recruitment stage. These include the PATHOS trial for HPV-positive oropharyngeal cancer (UK), the RTOG 1221 trial for HPVnegative oropharyngeal cancer (USA), and the ORATOR study for early-stage oropharyngeal cancer (Canada).

\section{The Future of TORS}

In addition to conducting RCTs, the next step in TORS involves the development of miniaturised, flexible robots, such as the i-Snake ${ }^{\circledR}$ (Imperial College London, London, UK) and Flex $^{\circledR}$ systems (Medrobotics ${ }^{\circledR}$, Raynham, MA, USA), which will be better-suited than the da Vinci ${ }^{\circledR}$ for otorhinolaryngology and head and neck surgery $[28,29]$. At the same time, multi- 
Garas and Arora : Robotic Head and Neck Surgery

Fig. 3. Upcoming surgical robots designed for head and neck surgery and their manufacturers.

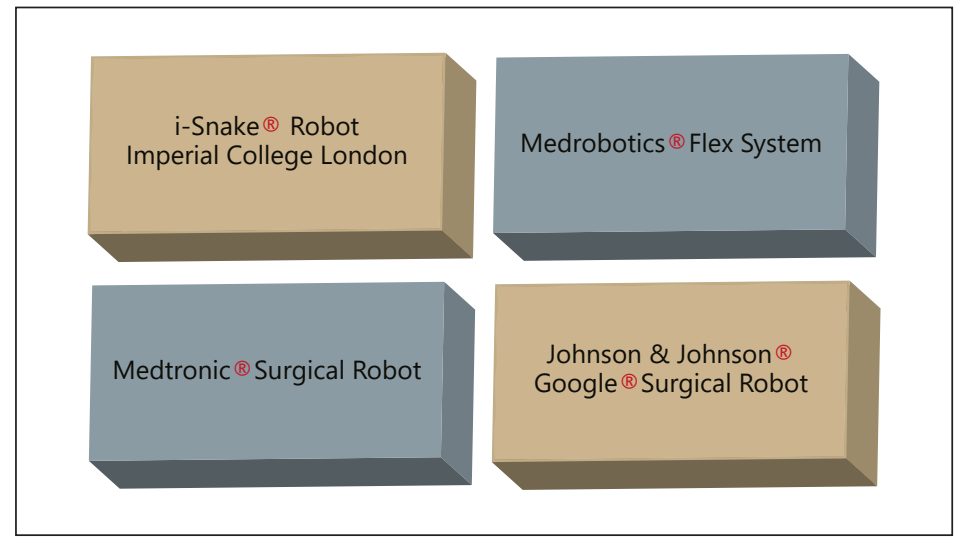

national medical device companies such as Medtronic, Inc. (Minneapolis, MN, USA) and Johnson \& Johnson, Inc. (New Brunswick, NJ, USA), the latter in collaboration with Google LLC (Mountain View, CA, USA), are also heavily investing in the design and manufacture of new surgical robots (Fig. 3). Their imminent market entry will introduce much awaited competition and can be expected to drive down costs, making robotic surgical technology (including TORS) more widely affordable whilst expanding the indications to, for example, tumours involving the nasopharynx, skull base, and those located in the glottic and subglottic larynx.

There are numerous other companies that have recently entered the robotic surgery market. Notable examples include Cambridge Medical Robotics, CMRSurgical,Ltd.(Cambridge, UK) with the Versius ${ }^{\circledR}$ surgical robotic system, TransEnterix, Inc. (Morrisville, NC, USA) with the Senhance ${ }^{\mathrm{TM}}$ multi-port and SurgiBot ${ }^{\mathrm{TM}}$ single-port robotic platforms, and Titan Medical, Inc. (Toronto, ON, Canada) with the SPORT ${ }^{\circledR}$ robot. Similar to the latest da Vinci ${ }^{\circledR}$ model (da Vinci Single-Site ${ }^{\circledR}$ ), these are nearly all single-port robotic systems. This characteristic, combined with the miniaturisation of the robotic arms, provides revolutionary anatomical access for NOTES including TORS.

The increasing demand for robotic surgery and its associated profitability, with the market worth just over USD 3 billion in 2014 and projected to exceed USD 20 billion by 2021, make it a very attractive area for investors [30]. This will ensure the development of newer, more advanced robotic systems, some of which will be designed specifically for minimally invasive head and neck surgery.

In terms of future directions, the introduction of augmented reality (AR) will facilitate image-guided TORS by providing the surgeon with real-time navigational cues and representations of key anatomical structures overlaid on the operative field. Other developments likely to follow include miniaturisation (nanorobots) and the development of autonomous surgical robots.

\section{Robotic Thyroid Surgery}

Thyroid nodules, including low-risk papillary thyroid cancer, are most commonly encountered in young women [31]. Naturally, this select patient group is the one most concerned about their cosmesis. This is more so in the Far East, where a horizontal neck scar is perceived to denote death [32].

The above concerns led to the development of "scarless-in-the-neck" endoscopic thyroidectomy. A variety of routes have been described over the years including the infraclavicular, 
Garas and Arora : Robotic Head and Neck Surgery

breast, axillary, axillo-bilateral-breast (ABBA), bilateral axillo-breast (BABA), and pastauricular and axillary (PAA) endoscopic approaches [33]. However, the multitude of endoscopic techniques trialed revealed none to be superior, as they were all characterised by the same limitations of endoscopic surgery; namely 2-dimensional (2-D) visualisation, restriction of instrument movement, the fulcrum effect, the need for $\mathrm{CO}_{2}$ insufflation, and dependence on multiple assistants [34].

Robotic technology, with its unique features including a dual-channel endoscope offering simultaneous image magnification and depth perception through 3-D visualisation combined with its wristed robotic instruments providing 7 degrees of freedom, has substantially enhanced surgical dexterity. Robotic surgery was originally introduced to overcome the limitations that characterise endoscopic surgery [34].

In 2009, a report on the first large series $(n=100)$ of transaxillary, gasless robotic thyroidectomy was published in South Korea [35]. The procedure proved to be popular among the local population and its uptake was rapid across the Far East, with thousands of cases performed over the subsequent years [36]. However, the uptake in the western world has been significantly lower and its value often questioned [37]. Various factors have been implicated including differences in culture, anthropometry, remuneration, as well as the presence of a National Thyroid Cancer Screening Programme in South Korea which has led to earlier detection of thyroid nodules, i.e., when they are much smaller (often $<1 \mathrm{~cm}$ ) and thus easier to resect [38].

\section{Robotic Parathyroid Surgery}

Following the wide uptake of robotic surgery by various surgical specialties and the increasing experience with endoscopic parathyroidectomy as well as robotic thyroidectomy, robotic parathyroidectomy was the natural next step [14, 38]. Robotic parathyroidectomy represents the "fourth generation" in the evolution of parathyroid surgery. The technique was first described by Tolley et al. [39] in 2011 in London, UK, and involves a scarless-in-theneck approach without the need for $\mathrm{CO}_{2}$ insufflation.

The preliminary results were promising, demonstrating robotic parathyroidectomy to represent a safe and feasible targeted parathyroidectomy technique in carefully selected patients [39]. Subsequent studies followed to reproduce the results and prospectively compare robotic parathyroidectomy to its minimally invasive open counterpart, illustrating the superiority of the robotic approach for early cosmesis and its equivalence in terms of cure rate for primary hyperparathyroidism [40]. However, the uptake of robotic parathyroidectomy remains limited due to a number of factors, including its niche role, high cost, technical complexity, and prolonged operative time.

\section{Conclusion}

Robotic head and neck surgery has transformed, in just over a decade, the management of benign and malignant diseases of the head and neck. The traditional morbid approaches for accessing and resecting tumours of the upper aerodigestive tract are often replaced with minimally invasive surgery in the form of TORS. The development of novel, flexible, miniaturised robots is likely to further expand the indications for TORS to tumours involving the nasopharynx and skull base as well as those located in the glottic and subglottic larynx, at the same time driving down costs as a result of market competition. 
Garas and Arora : Robotic Head and Neck Surgery

The situation for robotic thyroid and parathyroid surgery is less clear, as these procedures confer small value in a resource-limited health care system. They do, however, represent a viable option when a neck scar is undesirable for cultural or biological reasons. Until costs are sufficiently reduced, the role of these procedures is likely to remain in a niche capacity.

\section{Disclosure Statement}

Dr. George Garas, MD, FRCS holds a Royal College of Surgeons of England Doctoral Research Fellowship (grant No. GG 1037600/2017-2018) and is also supported by Imperial College London (grant No. CID 337755/2015-2018) and the Alexander S. Onassis Public Benefit Foundation (grant No. F ZM 014-1/20162017). The funders had no role in the study design, data collection and analysis, decision to publish, or preparation of the manuscript. All authors declare no conflicts of any commercial interest.

\section{References}

1 Kim KH, et al: Head and neck robotic surgery: pros and cons. Head Neck Oncol 2013;5:26.

2 Hockstein NG, et al: A history of robots: from science fiction to surgical robotics. J Robot Surg 2007;1:1:113118.

3 Capek K: Rossum's Universal Robots. New York, Penguin Group, 2004.

4 Gourin CG, Terris DJ: History of robotic surgery; in Faust RA (ed): Robotics in surgery: history, current and future applications. New York, Nova Science, 2006.

5 Unmanned Aircraft Systems DOD Purpose and Operational Use. http://www.defense.gov/UAS.

6 Kwoh YS, et al: A robot with improved absolute positioning accuracy for CT-guided stereotactic brain surgery. IEEE Trans Biomed Eng 1988;35:153-160.

7 Davies BL, et al: A surgeon robot prostatectomy - a laboratory evaluation. J Med Eng Technol 1989;13:237277.

8 Taylor RH, et al: Computer-integrated revision total hip replacement surgery: concept and preliminary results. Med Image Anal 1999;3:301-319.

9 Marescaux J, et al: Telerobotic laparoscopic cholecystectomy: initial clinical experience with 25 patients. Ann Surg 2001;234:1-7.

10 Badaan SR, Stoianovici D: robotic systems: past, present, and future; in Hemal AK, Menon M (eds): Robotics in Genitourinary Surgery. London, Springer, 2011, pp 655-665.

11 Trehan A, Dunn TJ: The robotic surgery monopoly is a poor deal. BMJ 2013;347:f7470.

12 da Vinci ${ }^{\circledR}$ Surgery: Minimally Invasive Surgery. http://www.davincisurgery.com.

13 Wong KA, et al: How can cardiothoracic and vascular medical devices stay in the market? Interact Cardiovasc Thorac Surg 2016;23:940-948.

14 Garas G, et al: Evidence-based surgery: barriers, solutions, and the role of evidence synthesis. World J Surg 2012;36:1723-1731.

15 O'Malley BW Jr, et al: Transoral robotic surgery (TORS) for base of tongue neoplasms. Laryngoscope 2006; 116:1465-1472.

16 Ansarin M, et al: Transoral robotic surgery vs. transoral laser microsurgery for resection of supraglottic cancer: a pilot surgery. Int J Med Robot 2014;10:107-112.

17 Arora A, et al: Determination of biometric measures to evaluate patient suitability for transoral robotic surgery. Head Neck 2015;37:1254-1260.

18 Weinstein GS, et al: Transoral robotic surgery for advanced oropharyngeal carcinoma. Arch Otolaryngol Head Neck Surg 2010;136:1079-1085.

19 Sims JR, et al: Transoral robotic medial hypopharyngectomy: surgical technique. Head Neck 2016;38:E2127E2129.

20 O'Malley BW Jr, et al: Transoral robotic surgery for parapharyngeal space tumors. ORL J Otorhinolaryngol Relat Spec 2010;72:332-336.

21 Mendelsohn $\mathrm{AH}$, et al: Outcomes following transoral robotic surgery: supraglottic laryngectomy. Laryngoscope 2013;123:208-214.

22 Mehta V, et al: A new paradigm for the diagnosis and management of unknown primary tumors of the head and neck: a role for transoral robotic surgery. Laryngoscope 2013;123:146-151.

23 Smith RV, et al: Transoral robotic total laryngectomy. Laryngoscope 2013;123:678-682.

24 Arora A, et al: Outcome of TORS to tongue base and epiglottis in patients with OSA intolerant of conventional treatment. Sleep Breath 2016;20:739-747.

25 Garas G, et al: Is transoral robotic surgery a safe and effective multilevel treatment for obstructive sleep apnoea in obese patients following failure of conventional treatment(s)? Ann Med Surg (Lond) 2017;19:55-61. 
Garas and Arora : Robotic Head and Neck Surgery

26 Georgalas C, et al: Assessment of obstruction level and selection of patients for obstructive sleep apnea surgery: an evidence-based approach. J Laryngol Otol 2010;124:1-9.

27 Van Abel KM, Moore EJ: The rise of transoral robotic surgery in the head and neck: emerging applications. Expert Rev Anticancer Ther 2012;12:373-380.

28 Mandapathil M, et al: Transoral surgery for oropharyngeal tumors using the Medrobotics ${ }^{\circledR}$ Flex $^{\circledR}$ system - a case report. Int J Surg Case Rep 2015;10:173-175.

29 Sodergren $\mathrm{MH}$, et al: Natural orifice translumenal endoscopic surgery: critical appraisal of applications in clinical practice. Surg Endosc 2009;23:680-687.

30 WinterGreen Research Report, Lexington, MA, 2014.

31 Garas G, et al: Is there survival benefit from life-long follow-up after treatment for differentiated thyroid cancer? Int J Surg 2013;11:116-121.

32 Tan CT, et al: "Scarless" (in the neck) endoscopic thyroidectomy (SET): an evidence-based review of published techniques. World J Surg 2008;32:1349-1357.

33 Christakis I, et al: Minimally invasive endocrine (thyroid, parathyroid, adrenal) surgery; in Hawthorne FT (ed): Minimally Invasive Surgery: Evolution of Operative Techniques, Safety and Effectiveness and Long-Term Clinical Outcomes. New York, Nova Science, 2014, pp 1-66.

34 Moorthy K, et al: Dexterity enhancement with robotic surgery. Surg Endosc 2004;18:790-795.

35 Kang SW, et al: Robot-assisted endoscopic surgery for thyroid cancer: experience with the first 100 patients. Surg Endosc 2009;23:2399-2406.

36 Lee SG, et al: Long-term oncologic outcome of robotic versus open total thyroidectomy in PTC: a case-matched retrospective study. Surg Endosc 2016;30:3474-3479.

37 Garas G, et al: Network analysis of surgical innovation: measuring value and the virality of diffusion in robotic surgery. PLoS One 2017;12:e0183332.

38 Arora A, et al: Comparing transaxillary robotic thyroidectomy with conventional surgery in a UK population: a case control study. Int J Surg 2016;27:110-117.

39 Tolley N, et al: Robotic-assisted parathyroidectomy: a feasibility study. Otolaryngol Head Neck Surg 2011;144: 859-866.

40 Tolley N, et al: Long-term prospective evaluation comparing robotic parathyroidectomy with minimally invasive open parathyroidectomy for primary hyperparathyroidism. Head Neck 2016;38:E300-E306. 